[9] B. Chen and P. K. Willett, "On the optimality of the likelihood-ratio test for local sensor decision rules in the presence of nonideal channels," IEEE Trans. Inf. Theory, vol. 51, pp. 693-699, Feb. 2005.

[10] M. Kam, Q. Zhu, and W. S. Gray, "Optimal data fusion of correlated local decisions in multiple sensor detection systems," IEEE Trans. Aerosp. Electron. Syst., vol. 28, pp. 916-920, Jul. 1992.

[11] S. Boyd and L. Vandenberghe, Convex Optimization. Cambridge, U.K.: Cambridge Univ. Press, 2003.

[12] J. F. Sturm and S. Zhang, "On cones of nonnegative quadratic functions," Math. Oper. Res., vol. 28, no. 2, pp. 246-267, May 2003.

\section{On the Optimal Performance in Asymmetric Gaussian Wireless Sensor Networks With Fading}

\author{
Hamid Behroozi, Fady Alajaji, and Tamás Linder
}

\begin{abstract}
We study the estimation of a Gaussian source by a Gaussian wireless sensor network (WSN) where $L$ distributed sensors transmit noisy observations of the source through a fading Gaussian multiple access channel to a fusion center. In a recent work Gastpar, ["Uncoded transmission is exactly optimal for a Simple Gaussian Sensor Network," IEEE Trans. Inf. Theory, vol. 54, no. 11, pp. 5247-5251, Nov. 2008] showed that for a symmetric Gaussian WSN with no fading, uncoded (analog) transmission achieves the optimal performance theoretically attainable (OPTA). In this correspondence, we consider an asymmetric fading WSN in which the sensors have differing noise and transmission powers. We first present lower and upper bounds on the system's OPTA under random fading. We next focus on asymmetric networks with deterministic fading. By comparing the obtained lower and upper OPTA bounds under deterministic fading, we provide a sufficient condition for the optimality of the uncoded transmission scheme for a given power tuple $P=\left(P_{1}, P_{2}, \ldots, P_{L}\right)$. Then, allowing the sensor powers to vary under a weighted sum constraint (this includes the sum-power constraint as a special case), we obtain a sufficient condition for the optimality of uncoded transmission and provide the system's corresponding OPTA.
\end{abstract}

Index Terms-Gaussian multiple access channel with fading, joint source-channel coding, power-distortion tradeoff, remote source coding, sensor networks, uncoded transmission.

\section{INTRODUCTION}

We consider the estimation of a memoryless Gaussian source by a Gaussian wireless sensor network (WSN) where $L$ sensors observe the source signal $X$ corrupted by additive independent noise. The overall

Manuscript received June 28, 2009; accepted December 19, 2009. First published January 15, 2010; current version published March 10, 2010. The associate editor coordinating the review of this manuscript and approving it for publication was Prof. Huaiyu Dai. This work was supported in part by a Postdoctoral Fellowship from the Ontario Ministry of Research and Innovation (MRI) and by the Natural Sciences and Engineering Research Council (NSERC) of Canada. The material in this correspondence was presented in part at the IEEE International Symposium on Information Theory (ISIT), Toronto, ON, Canada, July 2008, and at the IEEE Canadian Workshop on Information Theory (CWIT), Ottawa, ON, Canada, May 2009.

H. Behroozi was with the Department of Mathematics and Statistics, Queen's University, Kingston, ON, Canada. He is now with the Electrical Engineering Department, Sharif University of Technology, Tehran, Iran (e-mail: behroozi@sharif.edu).

F. Alajaji and T. Linder are with the Department of Mathematics and Statistics, Queen's University, Kingston, ON K7L 3N6, Canada (e-mail: fady@ @ast. queensu.ca; linder@mast.queensu.ca).

Color versions of one or more of the figures in this correspondence are available online at http://ieeexplore.ieee.org.

Digital Object Identifier 10.1109/TSP.2010.2040666

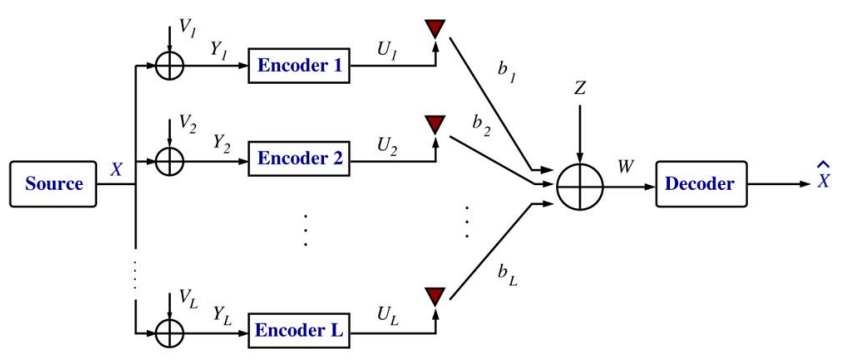

Fig. 1. A Gaussian wireless sensor network (WSN) with fading.

system is depicted in Fig. 1. The sensors communicate information about their observations through a fading Gaussian multiple access channel (MAC) to a single fusion center (FC). The fading coefficients are not known by the encoders but are available at the FC. The encoders are distributed and cannot cooperate to exploit the correlation between their inputs. Each encoder is subject to a transmission cost constraint. The FC aims to reconstruct the main source $X$ at the smallest cost in the communication link. Our interest lies in determining the optimal power-distortion region, with the fidelity of estimation at the FC measured by the mean squared-error (MSE) distortion. Specifically, for a given $L$-tuple of sensor powers $\boldsymbol{P}=\left(P_{1}, P_{2}, \ldots, P_{L}\right)$, we seek to determine the system's minimum achievable distortion which we refer to as the optimal performance theoretically attainable (OPTA).

In [1] and [2], it is proved that uncoded transmission is exactly optimal for symmetric Gaussian WSNs with a finite number of sensors and no fading. Uncoded transmission in this case (and in the rest of this correspondence) means scaling the encoder input subject to the channel power constraint and transmitting without explicit channel coding. Note that the separate source and channel coding theorem of Shannon [3] does not hold for this problem [1], [2]. In the case of deterministic fading, lower and upper bounds on the minimum distortion are presented in [1], [2], and [4], and for random fading, bounds are also presented in [4] and [5]. The minimum achievable distortion under a sum-power constraint for the uncoded transmission scheme in the WSN with deterministic fading is presented in [6]. The optimality of uncoded transmission in some other multiuser communication systems was recently shown in [7] and [8].

For the asymmetric fading Gaussian WSN, the following important issues remain unknown: Under either random or deterministic fading, what is the system's OPTA? Also, What is the optimal coding strategy that achieves OPTA? Our main contributions in this correspondence are as follows: First, by applying the idea of maximum correlation coefficient, illustrated in [9]-[11], we generalize the OPTA lower bound in [1] to an asymmetric Gaussian WSN with random fading. We show that the new bound is a tighter lower bound on the OPTA than that of [5] for a Gaussian WSN with random fading. We also analyze the uncoded transmission scheme and provide an upper bound on the OPTA for a given set of sensor powers. These two bounds constitute an extension of the bounds given for deterministic fading case in [1] and [2]. We next specialize the results to the case of deterministic fading. We establish a condition under which the lower and upper bounds on the system's OPTA coincide, hence making the uncoded transmission scheme optimal. We next allow the sensor powers to vary under a linear combination of powers (LCP) constraint. Aside from being a natural generalization of the sum-power constraint, the LCP constraint explicitly allows to introduce weight coefficients that reflect the potentially differing costs of supplying power to individual sensors. Our final contribution is to provide sufficient conditions for the optimality of un- 
coded transmission under a given LCP constraint and determine the system's corresponding OPTA.

The remainder of this correspondence is organized as follows. In Section II, we present the system model and problem statement. Section III provides lower and upper bounds on the OPTA in an asymmetric Gaussian WSN with random fading. In Section IV, we consider deterministic fading and provide matching conditions by comparing lower and upper bounds on the system's OPTA. For uncoded transmission, an optimal power allocation under an LCP constraint and a sufficient condition for its optimality are also obtained. Conclusions are presented in Section V.

\section{Problem Statement}

We consider a simple Gaussian WSN, illustrated in Fig. 1, where a team of $L$ sensors observe independent noisy versions of the memoryless Gaussian source $\{X[k]\}_{k=1}^{n}$. The underlying source $\{X[k]\}_{k=1}^{n}$ is a sequence of independent and identically distributed (i.i.d.) realvalued Gaussian random variables of mean zero and variance $\sigma_{X}^{2}$. For each observation time $k=1,2,3, \ldots$, the noisy observations are given by

$$
Y_{l}[k]=X[k]+V_{l}[k], \quad l=1, \ldots, L
$$

where $\left\{V_{l}[k]\right\}_{k=1}^{n}$ is a sequence of i.i.d. Gaussian random variables of mean zero and variance $\sigma_{V_{l}}^{2}$ which is independent of $\{X[k]\}_{k=1}^{n}$. We represent the first $n$ instances of $\{X[k]\}_{k \geq 1}$ and $\left\{Y_{l}[k]\right\}_{k>1}$ by the data sequences $X^{n}=(X[1], X[2], \ldots, X[n])$ and $Y_{l}^{n}=\left(\bar{Y}_{l}[1], Y_{l}[2], \ldots, Y_{l}[n]\right)$, respectively. The correlated sources $Y_{l}$ are not co-located and their observers cannot cooperate to directly exploit their correlation. Instead, the sequences $Y_{l}^{n}$ are separately encoded to $\varphi_{l}\left(Y_{l}^{n}\right)=U_{l}^{n}$ where the $\varphi_{l}$ are encoder functions in the form

$$
\varphi_{l}: \mathbb{R}^{n} \rightarrow \mathbb{R}^{n}, \quad l=1,2, \ldots, L .
$$

Each transmitted sequence $U_{l}^{n}$ is assumed to be average-power limited to $P_{l}$, i.e.,

$$
\frac{1}{n} \sum_{k=1}^{n} E\left[\left|U_{l}[k]\right|^{2}\right] \leq P_{l}, \quad l=1,2, \ldots, L .
$$

The sensors communicate the coded sequences to the decoder through a fading MAC. In fact, each transmitted signal $U_{l}$ is multiplied by a real-valued fading random variable $b_{l}, l=1,2, \ldots, L$. The $b_{l}$ are not known to the encoders but are available to the decoder. The fading coefficients have non-zero mean and are independent of each other and of the $U_{l}$ random variables. The time- $k$ output of the channel is given by

$$
W[k]=\sum_{l=1}^{L} b_{l}[k] U_{l}[k]+Z[k]
$$

where the channel noise $\{Z[k]\}_{k=1}^{n}$ is an i.i.d. sequence of Gaussian random variables of mean zero and variance $\sigma_{Z}^{2}$ that is independent of $X^{n}, V^{n}$, and the fading coefficients. Based on the channel output $W^{n}=(W[1], \ldots, W[n])$, the FC forms an estimate $\hat{X}^{n}$ of the main source $X^{n}$. Fidelity between $X^{n}$ and $\hat{X}^{n}$ is measured by the average squared error distortion, $\Delta=(1 / n) E\left[\sum_{j=1}^{n}(X[j]-\hat{X}[j])^{2}\right]$. The reconstructed signal can be described as $\hat{X}^{n}=\psi\left(W^{n}, \boldsymbol{b}^{n}\right)$, where the decoder function is a mapping

$$
\psi: \mathbb{R}^{n} \times \mathbb{R}^{n L} \rightarrow \mathbb{R}^{n} .
$$

Let $\mathcal{F}^{(n)}\left(P_{1}, P_{2}, \ldots, P_{L}\right)$ denote all encoder and decoder functions $\left(\varphi_{1}, \ldots, \varphi_{L}, \psi\right)$ that satisfy (2)-(5). For a particular coding scheme $\left(\varphi_{1}, \ldots, \varphi_{L}, \psi\right)$, the performance is determined by the cost vector $\boldsymbol{P}=\left(P_{1}, P_{2}, \ldots, P_{L}\right)$ and the incurred distortion $\Delta$. For any target distortion $D \geq 0$, the power-distortion region $\mathcal{P}(D)$ is defined as the convex closure of the set of all achievable power-distortion pairs $(\boldsymbol{P}, D)$, where a power-distortion pair $(\boldsymbol{P}, D)$ is achievable if for any $\delta>0$, there is an $n_{0}(\delta)$ such that for all $n \geq n_{0}(\delta)$ there exists $\left(\varphi_{1}, \ldots, \varphi_{L}, \psi\right) \in \mathcal{F}^{(n)}\left(P_{1}, P_{2}, \ldots, P_{L}\right)$ with distortion $\Delta \leq D+\delta$. Our aim is to investigate the power-distortion region of this fading Gaussian WSN and present lower and upper bounds on its OPTA, which is defined for a fixed $\boldsymbol{P}$ as

$$
D_{\min }(\boldsymbol{P})=\inf \{D \mid(\boldsymbol{P}, D) \in \mathcal{P}(D)\} .
$$

We also aim to derive optimality conditions for uncoded transmission in the sense of achieving the OPTA. In addition, we want to minimize the MSE distortion given a linear combination of powers (LCP) constraint, i.e.,

$$
\begin{cases}\text { minimize } & D(P) \\ \text { subject to } & \sum_{l=1}^{L} \beta_{l} P_{l} \leq \Omega\end{cases}
$$

where $D(\boldsymbol{P})$ is the distortion of the uncoded transmission scheme using power allocation $\boldsymbol{P}=\left(P_{1}, \ldots, P_{L}\right)$ and $\beta_{l}>0, l=1, \ldots, L$. This form of constraint is a slight generalization of the sum-power constraint which explicitly allows to introduce weight coefficients for the potentially differing costs of supplying power to individual sensors.

\section{INFORMATION-THEORETIC OPTA BOUNDS IN THE PRESENCE OF RANDOM FADING}

We present lower and upper bounds on the OPTA in an asymmetric Gaussian WSN with random fading. The lower bound is based on analyzing the remote source coding scenario, where the sensor observations are given to one common encoder; then applying the data processing as well as Jensen's inequalities and finally using the idea of maximum correlation coefficient, illustrated in [9]-[11]. The upper bound is based on analyzing uncoded transmission which is the transmission of scaled versions of the sensors observations.

\section{A. Lower Bound}

Proposition 1: A lower bound on the OPTA in an asymmetric Gaussian WSN with random fading is

$$
D_{\min } \geq D_{\ell} \triangleq D_{0}^{*}\left(1+\frac{\sigma_{X}^{2} \sigma_{Z}^{2} \sum_{l=1}^{L} \frac{1}{\sigma_{V_{l}}^{2}}}{\sigma_{Z}^{2}+\Omega_{r}}\right)
$$

where

$$
\begin{aligned}
D_{0}^{*}= & \left(\frac{1}{\sigma_{X}^{2}}+\sum_{l=1}^{L} \frac{1}{\sigma_{V_{l}}^{2}}\right)^{-1} \\
\Omega_{r}= & \sum_{l=1}^{L} P_{l} E\left[\left|b_{l}\right|^{2}\right] \\
& +2 \sigma_{X}^{2} \sum_{l=1}^{L} \sum_{j>l}^{L} E\left[b_{l}\right] E\left[b_{j}\right] \sqrt{\frac{P_{l} P_{j}}{\left(\sigma_{X}^{2}+\sigma_{V_{l}}^{2}\right)\left(\sigma_{X}^{2}+\sigma_{V_{j}}^{2}\right)}}
\end{aligned}
$$

and the expectations are taken with respect to the distribution of the fading random variables $b_{l}$.

Proof: See the proof for [4, Theorem 1]. This bound is an extension of [1, Theorem 4] to the random fading case.

Next we compare the bound of Proposition 1 with the lower bound presented in [5] and demonstrate that our bound is strictly tighter as 
long as at least one of the sensor observation noise variances $\sigma_{V_{l}}^{2}$ is nonzero.

The lower bound presented in [5], which is for the case where the sensors observations have the same noise level $\sigma_{V_{1}}^{2}=\cdots=\sigma_{V_{L}}^{2}=$ $\sigma_{V}^{2}$, can be expressed for a general asymmetric WSN as

$$
D_{\text {lower }}\left(L, P_{\text {tot }}\right)=D_{0}^{*}\left(1+\frac{\sigma_{X}^{2} \sigma_{Z}^{2} \sum_{l=1}^{L} \frac{1}{\sigma_{V_{l}}^{2}}}{\sigma_{Z}^{2}+P_{\text {tot }} \sum_{l=1}^{L} E\left[\left|b_{l}\right|^{2}\right]}\right)
$$

where it is assumed that there is a total power constraint in the communication channel, i.e., $1 / n \sum_{k=1}^{n} \sum_{l=1}^{L} E\left[\left|U_{l}[k]\right|^{2}\right] \leq P_{\text {tot }}$, and $P_{\text {tot }}$ denotes the average total sensor power available per observation vector $\left(U_{1}^{n}, \ldots, U_{L}^{n}\right)$. Let us denote

$$
\begin{aligned}
\sigma_{1}^{2}= & \sigma_{Z}^{2}+\sum_{l=1}^{L} P_{l} E\left[\left|b_{l}\right|^{2}\right] \\
& +2 \sigma_{X}^{2} \sum_{l=1}^{L} \sum_{j>l}^{L} E\left[b_{l}\right] E\left[b_{j}\right] \sqrt{\frac{P_{l} P_{j}}{\left(\sigma_{X}^{2}+\sigma_{V_{l}}^{2}\right)\left(\sigma_{X}^{2}+\sigma_{V_{j}}^{2}\right)}}
\end{aligned}
$$

and

$$
\sigma_{2}^{2}=\sigma_{Z}^{2}+P_{\text {tot }} \sum_{l=1}^{L} E\left[\left|b_{l}\right|^{2}\right]
$$

where $P_{\text {tot }}=\sum_{l=1}^{L} P_{l}$. By comparing (8) and (11), we observe that the only difference is in the denominator. The lower bound of (8) is in the form $D_{\ell}=D_{0}^{*}\left(1+\left(\sigma_{X}^{2} \sigma_{Z}^{2} \sum_{l=1}^{L}\left(1 / \sigma_{V_{l}}^{2}\right)\right) / \sigma_{1}^{2}\right)$ while $D_{\text {lower }}\left(L, P_{\text {tot }}\right)=D_{0}^{*}\left(1+\left(\sigma_{X}^{2} \sigma_{Z}^{2} \sum_{l=1}^{L}\left(1 / \sigma_{V_{l}}^{2}\right)\right) / \sigma_{2}^{2}\right)$. We want to show that $\sigma_{1}^{2} \leq \sigma_{2}^{2}$. Since the $\sigma_{V_{l}}^{2}$ are non-negative, we have

$$
\begin{aligned}
2 \sigma_{X}^{2} \sum_{l=1}^{L} \sum_{j>l}^{L} E\left[b_{l}\right] E\left[b_{j}\right] \sqrt{\frac{P_{l} P_{j}}{\left(\sigma_{X}^{2}+\sigma_{V_{l}}^{2}\right)\left(\sigma_{X}^{2}+\sigma_{V_{j}}^{2}\right)}} \\
\leq 2 \sum_{l=1}^{L} \sum_{j>l}^{L}\left|E\left[b_{l}\right] E\left[b_{j}\right]\right| \sqrt{P_{l} P_{j}} .
\end{aligned}
$$

Thus,

$$
\begin{aligned}
\sigma_{2}^{2}-\sigma_{1}^{2} \geq & P_{\text {tot }} \sum_{l=1}^{L} E\left[\left|b_{l}\right|^{2}\right]-\sum_{l=1}^{L} P_{l} E\left[\left|b_{l}\right|^{2}\right] \\
& -2 \sum_{l=1}^{L} \sum_{j>l}^{L}\left|E\left[b_{l}\right] E\left[b_{j}\right]\right| \sqrt{P_{l} P_{j}} \\
= & \sum_{j=1}^{L} \sum_{l=1}^{L} P_{j} E\left[\left|b_{l}\right|^{2}\right]-\sum_{l=1}^{L} P_{l} E\left[\left|b_{l}\right|^{2}\right] \\
& -2 \sum_{l=1}^{L} \sum_{j>l}^{L}\left|E\left[b_{l}\right] E\left[b_{j}\right]\right| \sqrt{P_{l} P_{j}} \\
= & \sum_{l=1}^{L} \sum_{j>l}^{L}\left(P_{l} E\left[\left|b_{j}\right|^{2}\right]+P_{j} E\left[\left|b_{l}\right|^{2}\right]\right) \\
& -2 \sum_{l=1}^{L} \sum_{j>l}^{L}\left|E\left[b_{l}\right] E\left[b_{j}\right]\right| \sqrt{P_{l} P_{j}} .
\end{aligned}
$$

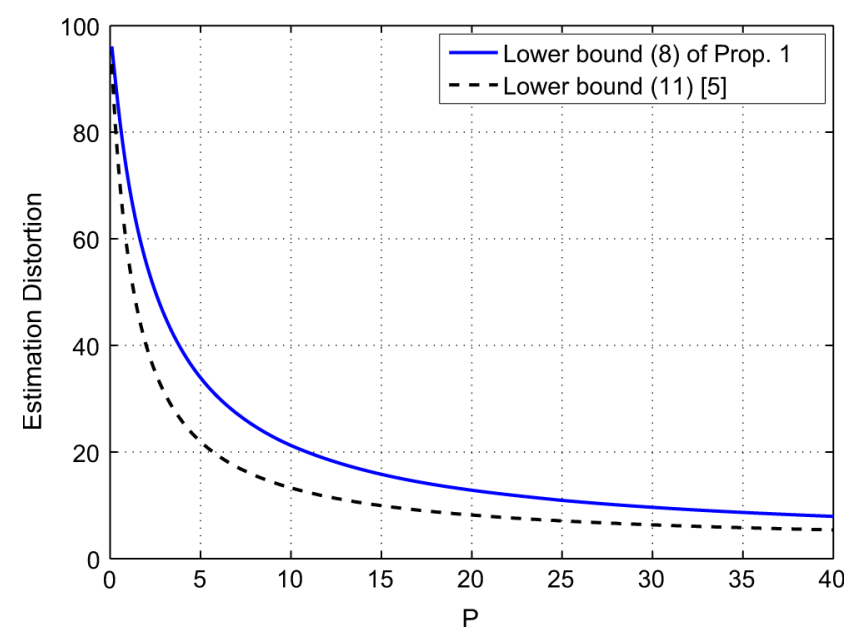

Fig. 2. The estimation distortion versus the transmission power, $P_{1}=P_{2}=$ $P$. We assume that $\sigma_{V_{1}}^{2}=\sigma_{V_{2}}^{2}=5, \sigma_{X}^{2}=100$ and $\sigma_{Z}^{2}=30$.

We have

$$
\begin{aligned}
\frac{1}{2}\left(P_{l} E\left[\left|b_{j}\right|^{2}\right]+P_{j} E\left[\left|b_{l}\right|^{2}\right]\right) & \geq \sqrt{P_{l} P_{j} E\left[\left|b_{l}\right|^{2}\right] E\left[\left|b_{j}\right|^{2}\right]} \\
& \geq\left|E\left[b_{l}\right] E\left[b_{j}\right]\right| \sqrt{P_{l} P_{j}}
\end{aligned}
$$

where the first inequality is due to the arithmetic-geometric mean inequality and the second inequality follows from the Cauchy-Schwarz inequality. Thus, a term-by-term comparison in (13) shows that the difference is non-negative, proving that $\sigma_{2}^{2} \geq \sigma_{1}^{2}$. Moreover, the inequality in (12) implies that $\sigma_{2}^{2}>\sigma_{1}^{2}$ as long as at least one of the $\sigma_{V_{l}}^{2}$ is positive. Therefore, the lower bound of (8) on $D_{\min }$ is tighter than (11); the improvement is mainly due to the maximization of the correlation coefficients.

1) Numerical Examples:

1) We consider a symmetric WSN consisting of two sensors and evaluate the resulting lower bounds numerically. We assume that $E\left[\left|b_{1}\right|^{2}\right]=E\left[\left|b_{2}\right|^{2}\right]=4, E\left[b_{1}\right]=E\left[b_{2}\right]=1$. The estimation distortion bound is plotted as a function of the power level $P_{1}=P_{2}=P$ in Fig. 2 . We observe that the distortion is a decreasing function of $P$ and that the lower bound $D_{\ell}$ of (8) is tighter than the lower bound $D_{\text {lower }}$ of (11). Specifically, if we calculate the percentage of the relative distortion gap (i.e., $\left(D_{\ell}-D_{\text {lower }}\right) / D_{\text {lower }} \%$ ) versus the value of $P$, we observe that $D_{\ell}$ performs $20 \%$ better (tighter) than $D_{\text {lower }}$ for $P>1.5$.

2) Now, we assume that the channel is not symmetric and plot the distortion bounds as a function of the power level $P_{1}=P_{2}=P$ in Fig. 3 for two different fading parameters:

$$
\begin{aligned}
& -E\left[\left|b_{1}\right|^{2}\right]=8, E\left[\left|b_{2}\right|^{2}\right]=4, E\left[b_{1}\right]=0.2, E\left[b_{2}\right]=1.5 \\
& -E\left[\left|b_{1}\right|^{2}\right]=35, E\left[\left|b_{2}\right|^{2}\right]=20, E\left[b_{1}\right]=2, E\left[b_{2}\right]=4 .
\end{aligned}
$$

Again, we observe that the lower bound $D_{\ell}$ is tighter than the lower bound $D_{\text {lower }}$.

3) We next consider an asymmetric WSN. We assume that $\sigma_{V_{2}}^{2}=$ $\sigma_{V_{1}}^{2}+\gamma, P_{1}=P_{2}=20, E\left[\left|b_{1}\right|^{2}\right]=E\left[\left|b_{2}\right|^{2}\right]=4$, and $E\left[b_{1}^{2}\right]=$ $E\left[b_{2}\right]=1$ and plot the estimation distortion bounds as a function of $\gamma$ in Fig. 4. There is almost a fixed gap between $D_{\ell}$ and $D_{\text {lower }}$. 


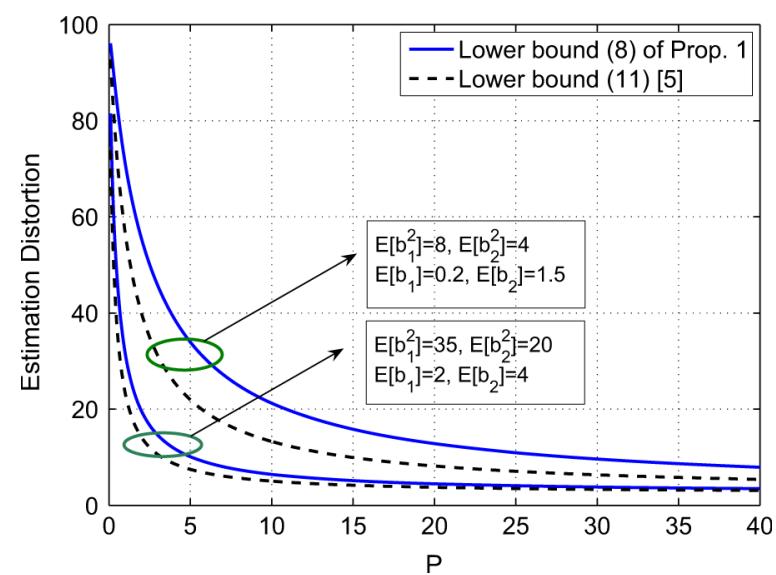

Fig. 3. The estimation distortion versus the transmission power, $P_{1}=P_{2}=P$ in a symmetric WSN with asymmetric fading MAC. We assume that $\sigma_{V_{1}}^{2}=$ $\sigma_{V_{2}}^{2}=5, \sigma_{X}^{2}=100$ and $\sigma_{Z}^{2}=30$.

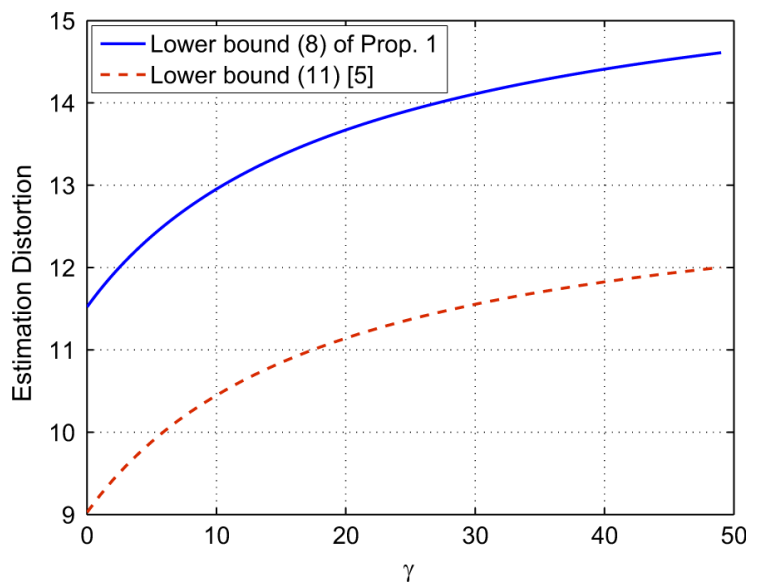

Fig. 4. The estimation distortion versus the value of $\gamma=\sigma_{V_{2}}^{2}-\sigma_{V_{1}}^{2}$. We assume that $P_{1}=P_{2}=20, \sigma_{V_{1}}^{2}=10, \sigma_{X}^{2}=100$ and $\sigma_{Z}^{2}=15$.

\section{B. Upper Bound: Analyzing Uncoded Transmission}

By analyzing the uncoded transmission in our Gaussian WSN, we next present an upper bound on the OPTA. In this approach, each sensor transmits its observation by simply scaling it to its power constraint, i.e., $U_{l}[k]=\sqrt{P_{l} /\left(\sigma_{X}^{2}+\sigma_{V_{l}}^{2}\right)} Y_{l}[k]$. The received signal at the $\mathrm{FC}$ is then given by

$$
W[k]=\sum_{l=1}^{L}\left\{\sqrt{\frac{P_{l}}{\sigma_{X}^{2}+\sigma_{V_{l}}^{2}}} b_{l}[k]\left(X[k]+V_{l}[k]\right)\right\}+Z[k] .
$$

Since the encoding is memoryless, the optimal (minimum mean-squared error) estimator is easy to obtain. By evaluating the resulting MSE distortion, we obtain an upper bound on the minimum achievable distortion, which is summarized in the next lemma.

Lemma 1: An upper bound on the OPTA in a Gaussian WSN with random fading can be expressed as

$$
D_{\text {min }} \leq D_{\text {uncoded }} \triangleq \sigma_{X}^{2} E\left(\frac{\sigma_{Z}^{2}+\sum_{l=1}^{L} \frac{P_{l} \sigma_{V_{l}}^{2}\left|b_{l}\right|^{2}}{\sigma_{X}^{2}+\sigma_{V_{l}}^{2}}}{\sigma_{Z}^{2}+\Omega_{r \mid b}}\right)
$$

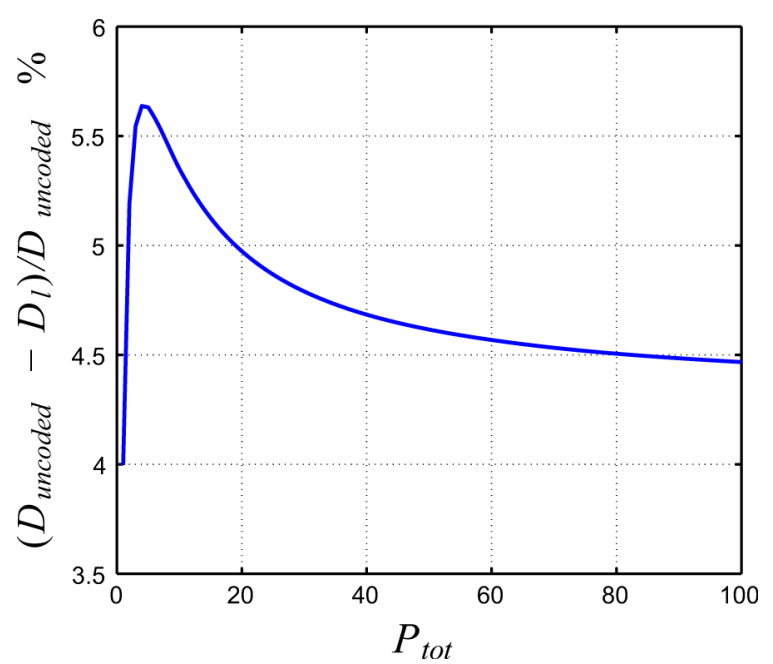

Fig. 5. Percentage of the relative distortion gap versus the sum-power $P_{\text {tot }}$. We assume that $L=2, \sigma_{X}^{2}=1, \sigma_{Z}^{2}=1, P_{1}=P_{2}$. The fading coefficients are taken as $k \times d^{-3.5}$, where $k$ is a normalization constant satisfying $E\left[b_{l}\right]=1$ and $d$ is drawn uniformly from the interval $[1,1.5]$.

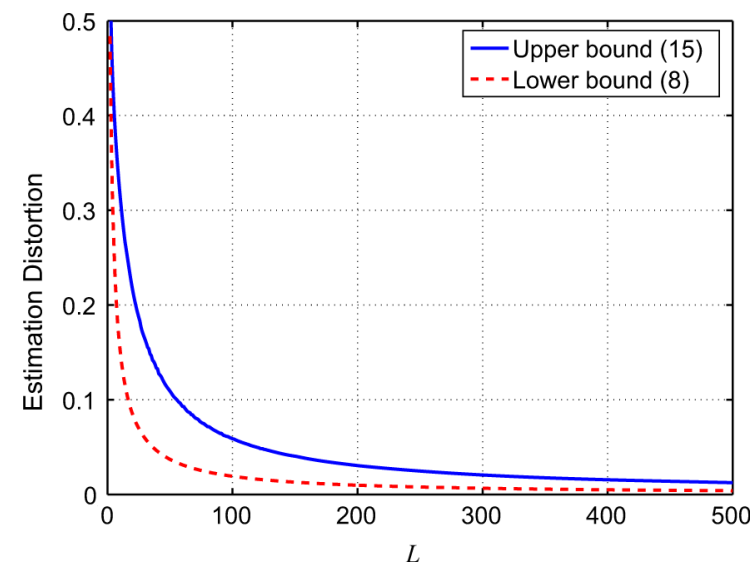

Fig. 6. The MSE estimation distortion versus the total number of sensors, $L$, under a uniform power schedule. The fading coefficients are taken as $k \times d^{-3.5}$, where $d$ is drawn uniformly from the interval $[1,6]$ and $k$ is a normalization constant satisfying $E\left[b_{l}\right]=1$. We assume that $P_{\text {tot }}=2, \sigma_{X}^{2}=1, \sigma_{Z}^{2}=1$, $\sigma_{V_{l}}^{2}=1($ for $l=1, \ldots, L)$.

where

$$
\Omega_{r \mid b}=2 \sigma_{X}^{2} \sum_{l=1}^{L} \sum_{j>l}^{L} b_{l} b_{j} \sqrt{\frac{P_{l} P_{j}}{\left(\sigma_{X}^{2}+\sigma_{V_{l}}^{2}\right)\left(\sigma_{X}^{2}+\sigma_{V_{j}}^{2}\right)}}+\sum_{l=1}^{L} P_{l}\left|b_{l}\right|^{2}
$$

and the expectation is with respect to the fading random variables, $b_{l}$.

In the following, we provide some examples in order to compare the lower and upper bounds numerically.

First, we consider a Gaussian WSN with $L=2$ sensors, where $\sigma_{V_{l}}^{2}=1$ (for $l=1, \ldots, L$ ). The fading coefficients of the channels are taken as $k \times d^{-3.5}$, where $k$ is a normalization constant chosen to satisfy $E\left[b_{l}\right]=1$ and $d$ is a random variable uniformly distributed on the interval $[1,1.5]$. In Fig. 5, we plot the percentage of the relative distortion gap (i.e., $\left(D_{\text {uncoded }}-D_{\ell}\right) / D_{\text {uncoded }} \%$ ) versus the sumpower $P_{\text {tot }}$ assuming a uniform power schedule. We note that the gap is less than $6 \%$ for all values of $P_{\text {tot }}$.

Fig. 6 shows the lower and upper bounds on the OPTA versus the total number of sensors, $L$ under a uniform power schedule and a sumpower $P_{\text {tot }}=2$. The fading coefficients are taken as $k \times d^{-3.5}$, where $d$ 
is drawn uniformly from the interval $[1,6]$ and $k$ is a normalization constant satisfying $E\left[b_{l}\right]=1$. We observe that the gap between the lower and upper bounds decreases as the total number of sensors increases.

Before closing this section, we point out that, using some algebraic manipulations, it is not hard to show that if $\sigma_{b_{l}}^{2}=0$, the lower bound of (8) and the upper bound of (14) agree under some conditions (see the proof of Corollary 1 in Section IV-A, presented in Appendix A). This means that uncoded transmission can achieve the optimal performance if the fading coefficients stay constant over the duration of transmission. This may apply to situations where the network conditions change very slowly. In the remainder of this work, we investigate the Gaussian WSN with such deterministic channel gains.

\section{DETERMINISTIC FADING SYSTEM}

\section{A. Optimality Condition for Uncoded Transmission}

Assume that the fading coefficients $b_{l}$ are fixed non-zero constants known at the fusion center. The lower bound of (8) and the upper bound of (14) can be expressed as follows:

$$
\begin{aligned}
& D_{\min } \geq D_{\ell}=D_{0}^{*}\left(1+\frac{\sigma_{X}^{2} \sigma_{Z}^{2} \sum_{l=1}^{L} \frac{1}{\sigma_{V_{l}}^{2}}}{\sigma_{Z}^{2}+\Omega_{r}}\right) \\
& D_{\text {min }} \leq D_{\text {uncoded }}=\left(\frac{\sigma_{X}^{2}}{\sigma_{Z}^{2}+\Omega_{r}}\right)\left(\sigma_{Z}^{2}+\sum_{l=1}^{L} \frac{P_{l} \sigma_{V_{l}}^{2}\left|b_{l}\right|^{2}}{\sigma_{X}^{2}+\sigma_{V_{l}}^{2}}\right)
\end{aligned}
$$

where

$\Omega_{r}=\sum_{l=1}^{L} P_{l} \sigma_{V_{l}}^{2}\left|b_{l}\right|^{2} /\left(\sigma_{X}^{2}+\sigma_{V_{l}}^{2}\right)+\left(\sum_{l=1}^{L} b_{l} \sqrt{P_{l} \sigma_{X}^{2} /\left(\sigma_{X}^{2}+\sigma_{V_{l}}^{2}\right)}\right)^{2}$.

In the symmetric case, where $\sigma_{V_{1}}^{2}=\cdots=\sigma_{V_{L}}^{2}=\sigma_{V}^{2}$ and $P_{1}=\cdots=$ $P_{L}=P$, the lower bound (16) and the upper bound (17) coincide if $b_{1}=\cdots=b_{L}=b$. Hence, we obtain the OPTA for the symmetric Gaussian WSN under deterministic and identical fading. This is the same result as recently established by Gastpar in [2] with $b_{1}=\cdots=$ $b_{L}=1$.

Corollary 1: For the asymmetric network with deterministic fading, the lower bound (16) and the upper bound (17) coincide if and only if

$$
b_{1} \sigma_{V_{1}}^{2} \sqrt{\frac{P_{1}}{\sigma_{X}^{2}+\sigma_{V_{1}}^{2}}}=\cdots=b_{L} \sigma_{V_{L}}^{2} \sqrt{\frac{P_{L}}{\sigma_{X}^{2}+\sigma_{V_{L}}^{2}}} .
$$

Proof: See Appendix A.

Hence, for a given set of powers, $\boldsymbol{P}$, if (18) is satisfied then the distortion achieved by the uncoded transmission is the smallest possible achievable distortion. Note that (18) is both necessary and sufficient for the upper and lower bounds to coincide, but it is only a sufficient condition for the optimality of uncoded transmission.

\section{B. Optimal Power Allocation for Uncoded Transmission Under an LCP Constraint}

In a WSN, many sensor devices are battery-powered and thus power constrained. Therefore, minimizing power consumption is critical in extending the lifetime of the individual sensor nodes and the entire network. A sum-power constraint has a direct impact on the network lifetime since it is imposed on the power consumption of all sensors taken together. However in some applications, in order to prevent excessive power consumption for individual sensors, or due to differing power supply capabilities at the sensors, individual power constraints for each sensor may also be desirable. This motivates us to combine cost coefficients with the sum-power constraint and consider a complexity constraint consisting of a an LCP. These cost coefficients might depend on the sensors' location and battery lifetime and can be assigned by the fusion center.

We thus herein consider an optimal power allocation for the uncoded transmission in order to minimize the MSE distortion under an LCP constraint, i.e.,

$$
\begin{cases}\text { minimize } & D(\boldsymbol{P}) \\ \text { subject to } & \sum_{l=1}^{L} \beta_{l} P_{l} \leq \Omega\end{cases}
$$

where $D(\boldsymbol{P})$ is the distortion of the uncoded transmission scheme using power allocation $\boldsymbol{P}=\left(P_{1}, \ldots, P_{L}\right)$ and $\beta_{l}>0, l=1, \ldots, L$.

In [6], by applying the Lagrange multiplier method, it was shown that the optimal distortion sum-power tradeoff $\left(D_{\text {uncoded }}, \sum_{l=1}^{L} P_{l}\right)$ for the uncoded scheme can be expressed as follows:

$$
D_{\text {uncoded }}\left(P_{\text {tot }}\right)=\left(\frac{1}{\sigma_{X}^{2}}+\sum_{l=1}^{L} \frac{1}{\sigma_{V_{l}}^{2}+\frac{\sigma_{Z}^{2}}{P_{\text {tot }} b_{l}^{2}}\left(\sigma_{X}^{2}+\sigma_{V_{l}}^{2}\right)}\right)^{-1}
$$

where $P_{\text {tot }}=\sum_{l=1}^{L} P_{l}$ is the sum-power. This tradeoff is also given in [2, eq. (6)], for the case of equal variance observation noises and equal channel gains. Since we want to compare lower and upper bounds on OPTA under an LCP constraint (which subsumes the sum-power constraint), we first need to provide closed form expressions of the optimal power allocation for the LCP case. The proof of the next lemma, which we omit, is based on an application of the Lagrange multiplier method. Note that as a special case, the lemma also yields the formula (20) for the minimum distortion under the sum-power constraint derived in [6].

Lemma 2 (Optimal Power Allocation for Uncoded Transmission under an LCP Constraint): An optimal power allocation scheme for the constraint $\Omega=\sum_{l=1}^{L} \beta_{l} P_{l}$ is given by

$$
P_{l}=\lambda_{l} \Omega=\frac{\left(\sigma_{X}^{2}+\sigma_{V_{l}}^{2}\right) \Omega}{\alpha b_{l}^{2}\left(\Omega \sigma_{V_{l}}^{2}+\sigma_{Z}^{2} \beta_{l}\left(\frac{\sigma_{X}^{2}+\sigma_{V_{l}}^{2}}{b_{l}^{2}}\right)\right)^{2}}, \quad l=1, \ldots, L
$$

where

$$
\alpha=\sum_{l=1}^{L} \frac{\beta_{l}\left(\sigma_{X}^{2}+\sigma_{V_{l}}^{2}\right)}{b_{l}^{2}\left(\Omega \sigma_{V_{l}}^{2}+\sigma_{Z}^{2} \beta_{l}\left(\frac{\sigma_{X}^{2}+\sigma_{V_{l}}^{2}}{b_{l}^{2}}\right)\right)^{2}} .
$$

Substituting the optimal $P_{l}$ given in (21) into (17) yields an upper bound on OPTA under the LCP constraint:

$$
D_{\text {uncoded }}(\Omega)=\left(\frac{1}{\sigma_{X}^{2}}+\sum_{l=1}^{L} \frac{1}{\sigma_{V_{l}}^{2}+\frac{\sigma_{Z}^{2} \beta_{l}}{b_{l}^{2} \Omega}\left(\sigma_{X}^{2}+\sigma_{V_{l}}^{2}\right)}\right)^{-1} .
$$

Corollary 2 (Uncoded Transmission Optimality Conditions Under an LCP Constraint): In an asymmetric Gaussian WSN with deterministic fading, uncoded transmission with the optimal power allocation given in (21) is optimal in the sense of achieving the OPTA under an LCP constraint if the following conditions hold

$$
b_{1} \sqrt{\frac{\sigma_{V_{1}}^{2}}{\beta_{1}\left(\sigma_{X}^{2}+\sigma_{V_{1}}^{2}\right)}}=\cdots=b_{L} \sqrt{\frac{\sigma_{V_{L}}^{2}}{\beta_{L}\left(\sigma_{X}^{2}+\sigma_{V_{L}}^{2}\right)}} .
$$

Proof: See Appendix B.

\section{CONCLUSION}

We considered a distributed WSN where $L$ noisy observations of a memoryless Gaussian source are transmitted through a fading Gaussian MAC to a decoder. The decoder wants to reconstruct the 
main source with an average distortion $D$ at the smallest possible power consumption in the communication link. Our goal was to characterize the power-distortion region achievable by any coding strategy regardless of delay and complexity. We obtained a lower bound on the system's OPTA, i.e., its minimum achievable distortion for a given set of powers $\left(P_{1}, P_{2}, \ldots, P_{L}\right)$. Also, by analyzing the uncoded transmission scheme, we provided an upper bound on the OPTA. When specialized to the network with deterministic fading, we provided sufficient conditions for the optimality of the uncoded transmission. We also obtained an optimal power allocation in order to minimize distortion for a given LCP constraint as well as an explicit condition for the optimality of uncoded transmission under an LCP constraint.

\section{APPENDIX A \\ PROOF OF COROLLARY 1}

Proof: We first rewrite the lower bound in (16) as

$$
D_{\ell}=\frac{\sigma_{X}^{2} \sigma_{Z}^{2}+D_{0}^{*} \sum_{l=1}^{L} \frac{P_{l} \sigma_{V_{l}}^{2}\left|b_{l}\right|^{2}}{\sigma_{X}^{2}+\sigma_{V_{l}}^{2}}+D_{0}^{*}\left(\sum_{l=1}^{L} b_{l} \sqrt{\frac{P_{l} \sigma_{X}^{2}}{\sigma_{X}^{2}+\sigma_{V_{l}}^{2}}}\right)^{2}}{\sigma_{Z}^{2}+\sum_{l=1}^{L} \frac{P_{l} \sigma_{V_{l}}^{2}\left|b_{l}\right|^{2}}{\sigma_{X}^{2}+\sigma_{V_{l}}^{2}}+\left(\sum_{l=1}^{L} b_{l} \sqrt{\frac{P_{l} \sigma_{X}^{2}}{\sigma_{X}^{2}+\sigma_{V_{l}}^{2}}}\right)^{2}} .
$$

Comparing (23) with the upper bound in (17) reveals that the lower bound and the upper bound coincide if and only if

$$
\left(\sigma_{X}^{2}-D_{0}^{*}\right) \sum_{l=1}^{L} \frac{P_{l} \sigma_{V_{l}}^{2}\left|b_{l}\right|^{2}}{\sigma_{X}^{2}+\sigma_{V_{l}}^{2}}=D_{0}^{*} \sigma_{X}^{2}\left(\sum_{l=1}^{L} b_{l} \sqrt{\frac{P_{l}}{\sigma_{X}^{2}+\sigma_{V_{l}}^{2}}}\right)^{2} .
$$

From the definition of $D_{0}^{*}$ we have $\left(\sigma_{X}^{2}-D_{0}^{*}\right) /\left(\sigma_{X}^{2} D_{0}^{*}\right)=$ $\sum_{l=1}^{L} 1 / \sigma_{V_{l}}^{2}$. As a result, the equality condition in (24) is equivalent to

$$
\left(\sum_{l=1}^{L} \frac{1}{\sigma_{V_{l}}^{2}}\right) \sum_{l=1}^{L} \frac{P_{l} \sigma_{V_{l}}^{2}\left|b_{l}\right|^{2}}{\sigma_{X}^{2}+\sigma_{V_{l}}^{2}}=\left(\sum_{l=1}^{L} b_{l} \sqrt{\frac{P_{l}}{\sigma_{X}^{2}+\sigma_{V_{l}}^{2}}}\right)^{2} .
$$

Setting $g_{l}=b_{l} \sigma_{V_{l}}^{2} \sqrt{P_{l} /\left(\sigma_{X}^{2}+\sigma_{V_{l}}^{2}\right)}$, we rewrite and simplify (25) as

$$
\sum_{l=1}^{L} \frac{g_{l}^{2}}{\sigma_{V_{l}}^{2}}\left(\sum_{j \neq l}^{L} \frac{1}{\sigma_{V_{j}}^{2}}\right)=2 \sum_{l=1}^{L} \sum_{j>l} \frac{g_{l} g_{j}}{\sigma_{V_{l}}^{2} \sigma_{V_{j}}^{2}}
$$

Comparing the coefficients of $1 /\left(\sigma_{V_{l}}^{2} \sigma_{V_{j}}^{2}\right)(l \neq j)$ on both sides of (26) shows that the equality holds if and only if $g_{l}^{2}+g_{j}^{2}=2 g_{l} g_{j}$; this is equivalent to $g_{l}=g_{j}$ which is our condition in (18).

\section{APPENDIX B}

\section{PROOF OF COROLLARY 2}

Proof: From the proof of Corollary 1, a necessary condition for the upper and the lower bound to coincide is that the equality in (25) holds. Substituting the optimal $P_{l}$ 's in this condition and setting $f_{l}=$ $\sigma_{V_{l}}^{2} /\left(\Omega \sigma_{V_{l}}^{2}+\sigma_{Z}^{2} \beta_{l}\left(\sigma_{X}^{2}+\sigma_{V_{l}}^{2}\right) / b_{l}^{2}\right)$, we obtain

$\sum_{l=1}^{L} \frac{f_{l}^{2}}{\sigma_{V_{l}}^{2}}\left(\sum_{j=1}^{L} \frac{1}{\sigma_{V_{j}}^{2}}\right)=\sum_{l=1}^{L} \frac{f_{l}^{2}}{\sigma_{V_{l}}^{2}}+2 \sum_{l=1}^{L} \sum_{j>l} \frac{f_{l} f_{j}}{\sigma_{V_{l}}^{2} \sigma_{V_{j}}^{2}} \operatorname{sgn}\left(b_{l}\right) \operatorname{sgn}\left(b_{j}\right)$ where $\operatorname{sgn}(x)=x /|x|$ is the sign of $x$. Subtracting $\sum_{l=1}^{L} f_{l}^{2} / \sigma_{V_{l}}^{2}$ from both sides in (27), we get

$$
\sum_{l=1}^{L} \frac{f_{l}^{2}}{\sigma_{V_{l}}^{2}}\left(\sum_{j \neq l}^{L} \frac{1}{\sigma_{V_{j}}^{2}}\right)=2 \sum_{l=1}^{L} \sum_{j>l} \frac{f_{l} f_{j}}{\sigma_{V_{l}}^{2} \sigma_{V_{j}}^{2}} \operatorname{sgn}\left(b_{l}\right) \operatorname{sgn}\left(b_{j}\right) .
$$

Comparing the coefficients of $1 /\left(\sigma_{V_{l}}^{2} \sigma_{V_{j}}^{2}\right)$ in both sides of (28) gives that the equality holds if and only if

$$
f_{l}^{2}+f_{j}^{2}=2 f_{l} f_{j} \operatorname{sgn}\left(b_{l}\right) \operatorname{sgn}\left(b_{j}\right) .
$$

Since $f_{l} \geq 0$, the equality holds if and only if $f_{l}=f_{j}$ and $\operatorname{sgn}\left(b_{l}\right) \operatorname{sgn}\left(b_{j}\right)=1$. Solving $f_{l}=f_{j}$ implies that

$$
\frac{\beta_{l}}{\sigma_{V_{l}}^{2}} \frac{\sigma_{X}^{2}+\sigma_{V_{l}}^{2}}{b_{l}^{2}}=\frac{\beta_{j}}{\sigma_{V_{j}}^{2}} \frac{\sigma_{X}^{2}+\sigma_{V_{j}}^{2}}{b_{j}^{2}} .
$$

Combining (29) with $\operatorname{sgn}\left(b_{l}\right) \operatorname{sgn}\left(b_{j}\right)=1$, we obtain (22) which completes the proof.

\section{REFERENCES}

[1] M. Gastpar, "Uncoded transmission is exactly optimal for a simple Gaussian sensor network," IEEE Trans. Inf. Theory, vol. 54, no. 11, pp. 5247-5251, Nov. 2008.

[2] M. Gastpar, "Uncoded transmission is exactly optimal for a simple Gaussian sensor network," presented at the 2007 Inf. Theory Applications Workshop, San Diego, CA, Jan. 2007.

[3] C. E. Shannon, "A mathematical theory of communication," Bell Syst. Tech. J., vol. 27, pp. 623-656, 1948.

[4] H. Behroozi, F. Alajaji, and T. Linder, "On the optimal power-distortion region for asymmetric Gaussian sensor networks with fading," presented at the IEEE ISIT, Toronto, ON, Canada, Jul. 2008.

[5] M. Gastpar and M. Vetterli, "Power, spatio-temporal bandwidth, and distortion in large sensor networks," IEEE J. Sel. Areas Commun., vol. 23, no. 4, pp. 745-754, Apr. 2005.

[6] J. Xiao, Z.-Q. Luo, S. Cui, and A. Goldsmith, "Power-efficient analog forwarding transmission in an inhomogeneous Gaussian sensor network," in Proc. 2005 IEEE 6th Workshop Signal Processing Advances Wireless Communications, Jun. 2005, pp. 121-125.

[7] A. Lapidoth and S. Tinguely, "Sending a bi-variate Gaussian source over a Gaussian MAC," in Proc. IEEE ISIT, Seattle, WA, Jul. 2006, pp. 2124-2128.

[8] S. Bross, A. Lapidoth, and S. Tinguely, "Broadcasting correlated Gaussians," presented at the IEEE ISIT, Toronto, ON, Canada, Jul. 2008.

[9] H. O. Hirschfeld, "A connection between correlation and contingency," Proc. Cambridge Philos. Soc., vol. 31, pp. 520-524, 1935.

[10] H. Gebelein, "Das statistische problem der korrelation als variations-und eigenwertproblem und sein zusammenhang mit der ausgleichungsrechnung," (in German) Z. Angew. Math. Mech., vol. 21 , pp. 364-379, 1941

[11] H. S. Witsenhausen, "On sequences of pairs of dependent random variables," SIAM J. Appl. Math., vol. 28, no. 1, pp. 100-113, Jan. 1975. 